\title{
Role of Spin-Dependent Terms in the Relationship among Nuclear Spin-Rotation and NMR Magnetic Shielding Tensors
}

\author{
3 I. Agustín Aucar, ${ }^{\dagger}$ Sergio S. Gomez, ${ }^{\dagger}$ Claudia G. Giribet, ${ }^{\ddagger}$ and Gustavo A. Aucar*,†] \\ $4{ }^{\dagger}$ Instituto de Modelado e Innovación Tecnológica, CONICET, and Departamento de Física, FCENA-UNNE, Avda. Libertad 5460, \\ 5 W3404AAS Corrientes, Argentina \\ $6{ }^{\star}$ Instituto de Física de Buenos Aires, CONICET, and Departamento de Física, FCEN-UBA, Ciudad Universitaria, C1428EGA Buenos \\ 7 Aires, Argentina
}

8 ABSTRACT: The broadly accepted procedure to obtain the experimental 9 absolute scale of NMR magnetic shieldings, $\sigma$, is well-known for nonheavy 10 atom-containing molecules. It was uncovered more than 40 years ago by the works 11 of Ramsey and Flygare. They found a quite accurate relationship among $\sigma$ and the 12 nuclear spin-rotation constants. Its relativistic extension was very recently 13 proposed, although it has an intrinsic weakness because a new SO-S two14 component term needs to be considered. We show how to overcome this 15 problem. We found that $\left(\boldsymbol{\nu}_{Y}^{\mathrm{S}}-\boldsymbol{\nu}_{Y}^{\text {atom,S }}\right)$ generalizes the SO-S term, where $\boldsymbol{\nu}_{Y}^{\mathrm{S}}=$ $16\left\langle\left\langle\left[\left(\left(\boldsymbol{r}-\boldsymbol{r}_{Y}\right) \times \boldsymbol{\alpha}\right) /\left(\left|\boldsymbol{r}-\boldsymbol{r}_{Y}\right|^{3}\right)\right] ; \boldsymbol{S}^{(4)}\right\rangle\right\rangle, \boldsymbol{r}-\boldsymbol{r}_{Y}$ is the electron position with respect to 17 the position of nucleus $Y$, and $S^{(4)}$ is the four-component total electron spin. When 18 including this new term, one finds that the best of our relativistic Flygare-like 19 models fits quite well with the results of the most accurate method available at the 20 moment. We also show that the difference among the parallel component of $21 \sigma(\mathrm{Xe})$ in $\mathrm{XeF}_{2}$ and $\sigma(\mathrm{Xe})$ of the free atom is almost completely described by that 22 new term.

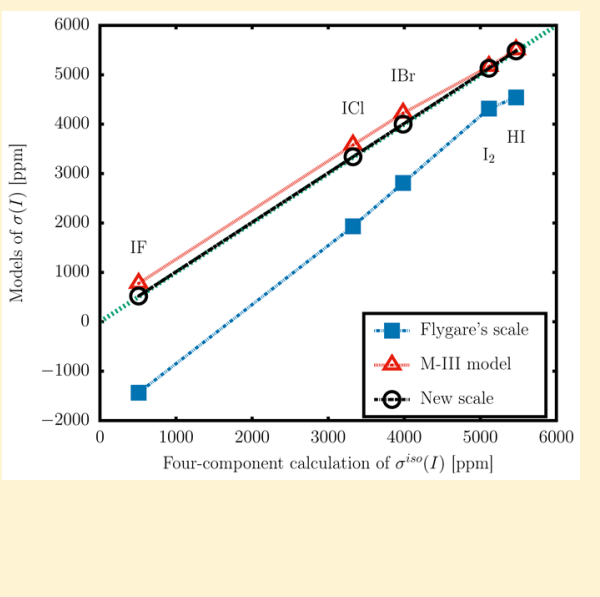

${ }_{23}^{23} \circlearrowleft$ ntil the past few years, one of the best ways to obtain by experiments the absolute value of the nuclear magnetic 25 shielding of a nucleus $Y, \sigma_{Y}$, was to apply the long-standing 26 Flygare's relationship. This situation changes dramatically 27 when the relativistic Dirac's theory of electrons is applied to 28 describe magnetic atomic and molecular properties. That 29 famous Flygare's relation is not valid any longer. The most 30 important deviations are found when heavy-atom-containing 31 molecules are considered, but they are also non-negligible in 32 molecular systems that contain only light atoms. Wasylishen 33 and coworkers had proven it experimentally by measuring the 34 parallel component of the shielding tensors of xenon 35 difluoride. $^{2}$

36 Many years were devoted to the search of new strategies that 37 could help to overcome this problem. The first important step 38 forward was the finding of the proper relativistic expression of 39 the nuclear spin-rotation tensor $(\boldsymbol{M})$, recently published by two 40 groups. ${ }^{3,4}$ Then, the next step shall be to look for a new 41 Flygare-type relationship that should be valid within the 42 relativistic framework. Even though, at first sight, a similar 43 relation among the relativistic spin-rotation (SR) and shielding 44 tensors seems difficult to obtain, few models were recently 45 proposed. $^{5-7}$ The most accurate of them has a two-component 46 spin-orbit (SO) term. ${ }^{7}$

47 The application of those models to linear molecules gave 48 results that are close to the current most exact values (obtained 49 from four-component methods) but not in all cases (see ref 7). 50 So, the new SO term seems to be one of few other unknown 51 terms that must be considered. Where shall we look to get better matching? Are there some more subtle physical reasons 52 for those differences? How do we go one step further in the 53 development of more accurate relativistic Flygare-type models? 54

We give some answers to these inquires. We shall show that 55 the SO term is the leading order contribution of the complete 56 spin-dependent (SD) contribution that must be included in 57 Flygare-type models to obtain more accurate results. 58

Ramsey first, and afterward Flygare, proposed a relationship 59 among $\boldsymbol{\sigma}_{Y}$ and $\boldsymbol{M}_{Y}$ tensors of a nucleus in a molecule, which 60 allows to indirectly obtain "experimental" values of absolute 61 shieldings using experimental SR constants ${ }^{1,8-10}$ together with 62 highly accurate calculations. This nonrelativistic (NR) relation 63 links the paramagnetic contribution to $\sigma_{Y}$ and the electronic 64 contribution to $\boldsymbol{M}_{Y}$ according to 65

$$
\begin{aligned}
\boldsymbol{\sigma}_{Y}^{\text {NR-para }} & =\frac{m_{\mathrm{p}}}{g_{Y}} \boldsymbol{M}_{Y}^{\text {NR-elec }} \otimes \boldsymbol{I} \\
& =\frac{m_{\mathrm{p}}}{g_{Y}}\left(\boldsymbol{M}_{Y}^{\mathrm{NR}}-\boldsymbol{M}_{Y}^{\mathrm{nuc}}\right) \otimes \boldsymbol{I}
\end{aligned}
$$

Atomic units were used in the last expression and shall be used 67 throughout this work, where $m_{\mathrm{p}}$ is the proton mass, $g_{\mathrm{Y}}$ is the 68 nuclear $g$-value of nucleus $Y$, and $\boldsymbol{I}$ is the tensor of molecular 69 moment of inertia with respect to the center of mass (CM) at 70

Received: October 11, 2016

Accepted: November 28, 2016

Published: November 28, 2016 
71 the equilibrium geometry. Equation 1 explicitly shows that the $72 \boldsymbol{M}_{Y}$ tensor can be expressed as the sum of two terms: one 73 electronic and one nuclear. The $\boldsymbol{M}_{Y}^{\mathrm{NR}-\text { elec }}$ tensor is the linear 74 response that takes account of two external potentials 75 originated in (i) the total electronic orbital angular momentum 76 centered in the molecular CM and (ii) the nuclear magnetic 77 moment of nucleus $Y$. Besides, eq 1 is valid only when the 78 gauge origin $(\mathrm{GO})$ of the magnetic potential is located at that 79 CM.

80 Another finding of Flygare and coworkers was a relationship 81 between the diamagnetic contribution to $\boldsymbol{\sigma}$ of a nucleus in a 82 molecule, $\sigma_{Y}^{\mathrm{NR}-\text { dia }}$, when the GO is placed at the nucleus position $83 Y$, and the shielding of that nucleus in the free atom, $\boldsymbol{\sigma}_{Y}^{\text {atom,NR }}$ 84 (which has only diamagnetic contributions within the NR 85 domain). They first found that $\boldsymbol{\sigma}_{Y}^{\mathrm{NR} \text {-dia }}$ can be approximated as 86 the sum of $\boldsymbol{\sigma}_{Y}^{\text {atom,NR }}$ and another tensor that only depends on the 87 nuclear positions at equilibrium (the first term in equation 6 of 88 ref 9 , which includes our $\boldsymbol{M}_{Y}^{\text {nuc }}$ as part of it; see ref 3). ${ }^{9,10}$ 89 Applying this relation, they found that the average (isotropic) 90 values are more accurate than the individual tensor elements. In 91 further works, they improved the last relation by using an atom 92 dipole model including two other terms: a dipole one, in 93 general quite small, and a quadrupole term. ${ }^{1,11}$

94 It is important to highlight here that although Flygare found 95 this relation taking the center of rotation and the GO positions 96 in the SR and shielding tensors (respectively) at the position of 97 nucleus $Y, 9$ it also happens that when the $\mathrm{CM}$ is considered as 98 the center of rotation for the SR tensor and also as GO for the 99 magnetic potential in the shielding tensor, Flygare's diamag100 netic approach can be expressed as (it can be shown following ${ }_{101}$ the arguments of ref 3) $\boldsymbol{\sigma}_{Y}^{\text {NR-dia }} \cong \boldsymbol{\sigma}_{Y}^{\text {atom,NR }}+\frac{m_{\mathrm{p}}}{g_{Y}} \boldsymbol{M}_{Y}^{\text {nuc }} \otimes \boldsymbol{I}$.

102 Because the Flygare's quadrupole term appears only for 103 individual tensor elements of $\boldsymbol{\sigma}_{Y}^{\mathrm{NR} \text {-dia }}$ (it does not contribute to 104 the isotropic diamagnetic shielding), ignoring this term may 105 introduce significant errors in the approximation of the tensor 106 elements of $\boldsymbol{\sigma}_{Y}^{\text {NR-dia }}$. So the isotropic diamagnetic shielding can 107 accurately be reproduced as the sum of $\sigma_{Y}^{\text {atom,NR }}$ and $M_{Y}^{\text {nuc-iso }}$ (in $108 \mathrm{ppm}$ ), whereas only fair estimations will be obtained for the 109 individual tensor elements if the quadrupole term is ignored. ${ }^{11}$

110 From these considerations, one obtains the well-known 111 Flygare's relationship

$$
\begin{aligned}
\boldsymbol{\sigma}_{Y} & =\boldsymbol{\sigma}_{Y}^{\mathrm{NR}-\mathrm{para}}+\boldsymbol{\sigma}_{Y}^{\mathrm{NR}-\mathrm{dia}} \\
& \approx \frac{m_{\mathrm{p}}}{g_{Y}} \boldsymbol{M}_{Y}^{\mathrm{NR}} \otimes \boldsymbol{I}+\boldsymbol{\sigma}_{Y}^{\text {atom,NR }}
\end{aligned}
$$

113 which is restricted to rigid rotor molecules in their equilibrium 114 geometry. The approximation is generally more accurate for the 115 isotropic than for each individual tensor element.

116 As mentioned above, few recent works have shown that such 117 a relationship is broken within the relativistic regime. ${ }^{3-6,12}$ In 118 our search for generalizing Flygare's NR relationship, we have 119 proposed three increasingly accurate relativistic theoretical 120 models. $^{7}$ Such models were related to a few previous ones, such 121 as those of Malkin and coworkers ${ }^{5}$ and Xiao and coauthors. ${ }^{6}$ 122 However, the aim of the present work is to go one important 123 step further.

124 The most accurate of the above-mentioned models is the M125 III one, which was defined as

$$
\boldsymbol{\sigma}_{Y}^{\mathrm{M}-\mathrm{III}}=\frac{m_{\mathrm{p}}}{g_{Y}} \boldsymbol{M}_{Y} \otimes \boldsymbol{I}+\boldsymbol{\sigma}_{Y}^{\text {atom }}+\frac{1}{2} \boldsymbol{\sigma}_{Y}^{\mathrm{SO}-\mathrm{S}}
$$

This model includes terms that are formally the same as those 127 of the original Flygare's relationship plus the new SO term. The 128 second term of eq 3 is the free atomic shielding, which includes 129 both its "paramagnetic-like" (e-e) and "diamagnetic-like" (e-p) 130 relativistic values. ${ }^{13}$ Again, isotropic values shall be more 131 accurate than the individual tensor elements due to Flygare's 132 approach to diamagnetic contributions.

One can expect that the model M-III shall fail when higher 134 order effects are not vanishingly small. So, a more accurate 135 model shall be obtained when the SO-S term is replaced by its 136 four-component counterpart.

In the special case of linear molecules, the first term of the 138 right-hand side of eq 3 will not have parallel components 139 (where the main line of the molecular geometry is parallel). In 140 ref 7, we studied the accuracy of the M-III model. With such an 141 aim we analyzed the (e-e) part of the perpendicular 142 components of $\boldsymbol{\sigma}_{Y}^{\mathrm{M}-\mathrm{III}}$ of eq 3 . We studied how close $\frac{1}{2} \boldsymbol{\sigma}_{\perp, Y}^{\mathrm{SO}-\mathrm{S}}{ }_{143}$ and $\boldsymbol{\sigma}_{\perp, Y}^{(\mathrm{e}-\mathrm{e})}-\frac{m_{\mathrm{p}} I}{g_{Y}} \boldsymbol{M}_{\perp, Y}^{(\mathrm{e}-\mathrm{e})}-\boldsymbol{\sigma}_{Y}^{\text {atom(e-e) }}$ are in a series of linear ${ }_{144}$ molecules. The last difference was coined $\Delta \sigma_{\perp, Y}^{\mathrm{II}}$ because it is 145 the difference between $\boldsymbol{\sigma}_{\perp, Y}^{(\mathrm{e}-\mathrm{e})}$ and $\boldsymbol{\sigma}_{\perp, Y}^{\mathrm{II}(\mathrm{e}-\mathrm{e})}$ (see ref 7 ). Such a 146 difference may be due to relativistic higher order terms, not 147 included in the SO-S one.

148

How do we get such terms? Let us start from the definition 149 of the relativistic electronic contribution to the SR tensor. ${ }^{3}$ It is 150 known that

$$
\begin{aligned}
\boldsymbol{M}_{Y}^{\mathrm{elec}}= & \boldsymbol{M}_{Y}^{\mathrm{L}}+\boldsymbol{M}_{Y}^{\mathrm{S}} \\
= & \frac{g_{Y}}{2 m_{\mathrm{p}} c}\left\langle\left\langle\frac{\left(\boldsymbol{r}-\boldsymbol{r}_{Y}\right) \times \boldsymbol{\alpha}}{\left|\boldsymbol{r}-\boldsymbol{r}_{Y}\right|^{3}} ;\left(\boldsymbol{r}-\boldsymbol{r}_{\mathrm{CM}}\right) \times \boldsymbol{p}\right\rangle\right\rangle \otimes \boldsymbol{I}^{-1} \\
& +\frac{g_{Y}}{2 m_{\mathrm{p}} c}\left\langle\left\langle\frac{\left(\boldsymbol{r}-\boldsymbol{r}_{Y}\right) \times \boldsymbol{\alpha}}{\left|\boldsymbol{r}-\boldsymbol{r}_{Y}\right|^{3}} ; \boldsymbol{S}^{(4)}\right\rangle\right\rangle \otimes \boldsymbol{I}^{-1} \\
= & \frac{g_{Y}}{m_{\mathrm{p}}}\left[\frac{1}{2 c}\left(\boldsymbol{\nu}_{Y}^{\mathrm{L}}+\boldsymbol{\nu}_{Y}^{\mathrm{S}}\right)\right] \otimes \boldsymbol{I}^{-1}
\end{aligned}
$$

where $\boldsymbol{r}-\boldsymbol{r}_{Y}$ are the electronic position operators and $\boldsymbol{\alpha}$ stands 153 for the Dirac matrices. The linear responses $\boldsymbol{\nu}_{Y}^{\mathrm{L}}$ and $\boldsymbol{\nu}_{Y}^{\mathrm{S}}$ are 154 associated with $\boldsymbol{M}_{Y}^{\mathrm{L}}$ and $\boldsymbol{M}_{Y}^{\mathrm{S}}$, respectively. The SO-S mechanism 155 arises from $\boldsymbol{M}_{Y}^{\mathrm{S}(\mathrm{e}-\mathrm{e})}$ ?

156

To generalize the SO-S term to a four-component 157 expression, the same methodology used previously in ref 14158 will be applied here. This scheme is related to the Dyall 159 formalism, ${ }^{15}$ where the Dirac-Coulomb-Breit Hamiltonian is 160 splitted into two Hamiltonians, that is, one that is spin-free 161 (SF) and another one that is SO-dependent. From this 162 separation, we are able to define a four-component SO 163 mechanism for $\boldsymbol{\sigma}_{Y}$

$$
\sigma_{Y}^{\mathrm{SD}}=\sigma_{Y}-\sigma_{Y}^{\mathrm{SF}}
$$

where $\boldsymbol{\sigma}_{Y}^{\mathrm{SF}}$ is the shielding tensor obtained when considering the 166 SF Hamiltonian.

A similar definition can be applied to the linear response $\boldsymbol{\nu}_{Y}^{\mathrm{S}} 168$ of the second term of eq 4 ,

$$
\boldsymbol{\nu}_{Y}^{\mathrm{S}-\mathrm{SD}}=\boldsymbol{\nu}_{Y}^{\mathrm{S}}-\boldsymbol{\nu}_{Y}^{\mathrm{S}-\mathrm{SF}}
$$

Replacing $\frac{1}{2} \boldsymbol{\sigma}_{Y}^{\text {SO-S }}$ with $\frac{1}{2 c} \boldsymbol{\nu}_{Y}^{\text {S-SD(e-e) }}$ in eq 3, we obtain 
172

$$
\boldsymbol{\sigma}_{Y}^{\mathrm{M}-\mathrm{IV}}=\frac{m_{\mathrm{p}}}{g_{Y}} \boldsymbol{M}_{Y} \otimes \boldsymbol{I}+\boldsymbol{\sigma}_{Y}^{\text {atom }}+\frac{1}{2 c} \boldsymbol{\nu}_{Y}^{\mathrm{S}-\mathrm{SD}(\mathrm{e}-\mathrm{e})}
$$

173 where only (e-e) rotations are considered in $\boldsymbol{\nu}_{Y}^{\text {S-SD }}$ because they 174 (and not the (e-p) ones) give rise to the SO-S term as a leading 175 order correction. ${ }^{16}$ We should also mention here that for linear 176 molecules the first term has null parallel component.

177 Now we shall compare the performance of M-III and M-IV 178 models on a set of linear molecules, $\mathrm{HX},(X=\mathrm{F}, \mathrm{Cl}, \mathrm{Br}, \mathrm{I}, \mathrm{At})$, $179 X \mathrm{~F}, \mathrm{LX}(X=\mathrm{Cl}, \mathrm{Br}, \mathrm{I})$, and $\mathrm{XeF}_{2}$, and see which one is the best. 180 Four-component calculations of both, $\boldsymbol{\sigma}_{Y}$ and $\boldsymbol{M}_{Y}$ tensor 181 elements were performed at the RPA level of approach of the 182 polarization propagator formalism with Dirac-Coulomb 183 Hartree-Fock wave functions, employing a developmental 184 version of the DIRAC14 program package. ${ }^{17}$ Gaussian-type 185 nuclear models were employed in all calculations.

186 To fulfill Flygare's prescription for the relation of eq 1, the $187 \mathrm{GO}$ was placed at the molecular CM for the calculation of $\boldsymbol{\sigma}_{Y}$. 188 Furthermore, to perform a four-component calculation of $\boldsymbol{\sigma}_{Y}^{\text {atom }}$, 189 an electron was added to the halogen free-atoms. Calculations 190 of open-shell structures are not available in the DIRAC code. 191 Experimental internuclear distances for molecules in their 192 gaseous phase were considered and taken from refs 18 (all 193 compounds but IF) and 19 (only IF). For HAt, an optimized 194 internuclear distance was used (1.720284558 $\AA$ ).

195 All calculations were performed using large uncontracted 196 Gaussian basis sets and the common gauge-origin (CGO) 197 approach. The NR Dunning's aug-cc-pCV5Z basis set was used 198 for hydrogen, fluorine, and chlorine atoms, ${ }^{20}$ whereas for $\mathrm{Br}, \mathrm{I}$, $199 \mathrm{Xe}$, and At, the dyall.acv4 $\mathrm{z}^{21}$ basis set was employed. In all cases 200 the restricted kinetic balance prescription (RKB) was used, 201 although calculations of few selected molecules were also 202 performed using the unrestricted kinetic balance prescription 203 (UKB). ${ }^{13}$ This is because to evaluate the performance of our 204 models, it is enough to consistently use RKB with a huge basis 205 set and also because the differences found applying UKB are 206 such that the accuracy of our models does not vary.

207 In Figure 1 we show the performance of SO-S and S-SD 208 contributions versus $\Delta \sigma_{\perp, Y}^{\mathrm{II}}$. It gives us an insight into how

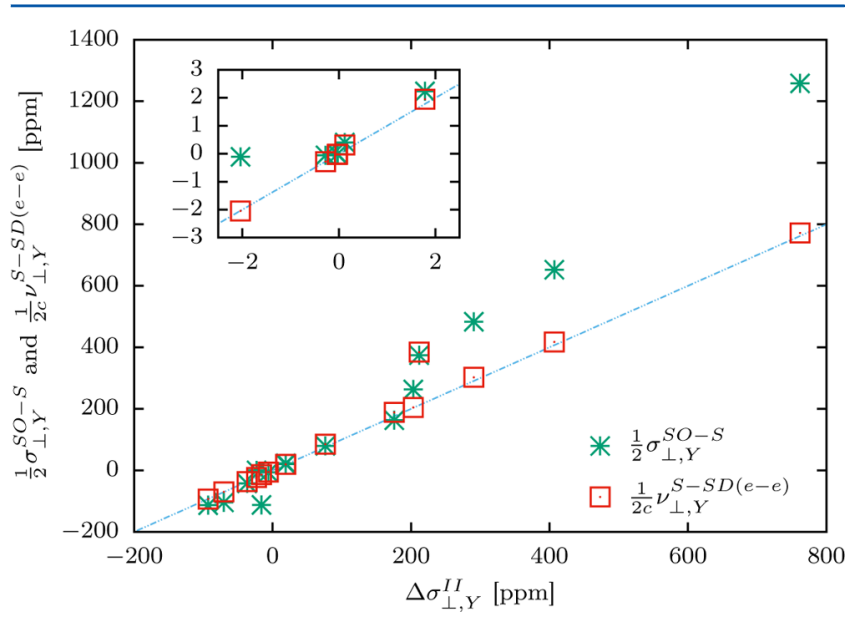

Figure 1. Comparison of the matching of $\frac{1}{2} \sigma_{\perp, Y}^{\mathrm{SO}-\mathrm{S}}$ and $\frac{1}{2 c} \nu_{\perp, Y}^{\mathrm{S}-\mathrm{SD}(\mathrm{e}-\mathrm{e})}$ with $\Delta \sigma_{\perp, Y}^{\mathrm{II}}$, where $Y$ represents each nucleus of all the compounds studied in the present work. The agreement between $\Delta \sigma_{\perp, Y}^{\mathrm{II}}$ and $\frac{1}{2 c} \nu_{\perp, Y}^{\mathrm{S}-\mathrm{SD}(\mathrm{e}-\mathrm{e})}$ is by far better than that with $\frac{1}{2} \sigma_{\perp, Y}^{\text {SO-S }}$ for all $Y$ in all compounds, except for At in HAt. important the S-SD term is. It also shows that the difference 209 between $\Delta \sigma_{\perp, Y}^{\mathrm{II}}$ and $\frac{1}{2} \sigma_{\perp, Y}^{\mathrm{SO} S}$ arises from higher order terms. 210

The replacement of $\frac{1}{2} \sigma_{\perp, Y}^{\mathrm{SO}-\mathrm{S}}$ by $\frac{1}{2 c} \nu_{\perp, Y}^{\mathrm{S}-\mathrm{SD}(\mathrm{e}-\mathrm{e})}$ greatly improves 211 the behavior of the M-III model. The subtraction between 212 $\frac{1}{2 c} \nu_{\perp, Y}^{\mathrm{S}-\mathrm{SD}(\mathrm{e}-\mathrm{e})}$ and $\Delta \sigma_{\perp, Y}^{\mathrm{II}}$ has nearly the same value for each nucleus $Y$ in different molecules. Such a subtraction is close (in 213 ppm) to 0.1 when $Y=\mathrm{F}, 0.2$ for $\mathrm{Cl}, 1$ for $\mathrm{Br}, 9$ for I, and 172 for 214 At. So, the remaining difference seem to be of atomic nature. 215 We then evaluated the term $\frac{1}{2 c} \nu_{Y^{-}}^{\text {atom,S-SD(e-e) }}$ and found the ${ }_{216}$ following values (in ppm): 0.0029 for $\mathrm{X}=\mathrm{F}, 0.0299$ for $\mathrm{X}=\mathrm{Cl}, 217$ 0.9324 for $\mathrm{X}=\mathrm{Br}, 8.2424$ for $\mathrm{X}=\mathrm{I}, 1.1477$ for $\mathrm{X}=\mathrm{Xe}$, and 218 171.7071 for $\mathrm{X}=$ At. They have a remarkable similarity with 219 those given above.

There is an error that comes from the fact that we have 221 calculated $\sigma^{\text {atom(e-e) }}\left(Y^{-}\right)$instead of $\sigma^{\text {atom(e-e) }}(Y)$ (see Table 1$)$. In $222 \mathrm{t} 1$ all cases, $\sigma^{\text {atom(e-e) }}\left(Y^{-}\right)$are larger by few $\mathrm{ppm}$ than the values of 223 $\sigma_{\perp, Y}^{(\mathrm{e}-\mathrm{e})}-\frac{m_{\mathrm{p}} I}{g_{Y}} M_{\perp, Y}^{(\mathrm{e}-\mathrm{e})}-\frac{1}{2 c} \nu_{\perp, Y}^{\mathrm{S}-\mathrm{SD}(\mathrm{e}-\mathrm{e})}$ and $\sigma_{\|, Y}^{(\mathrm{e}-\mathrm{e})}-\frac{1}{2 c} \nu_{\|, Y}^{\mathrm{S}-\mathrm{SD}(\mathrm{e}-\mathrm{e})}$. The worst results were found for At, where the parallel and 224 perpendicular com ponent s of 225 $\boldsymbol{\sigma}_{\mathrm{At}}^{(\mathrm{e}-\mathrm{e})}-\frac{m_{\mathrm{p}}}{g_{Y}} \boldsymbol{M}_{\mathrm{At}}^{(\mathrm{e}-\mathrm{e})} \otimes \boldsymbol{I}-\frac{1}{2 c} \boldsymbol{\nu}_{\mathrm{At}}^{\mathrm{S}-\mathrm{SD}(\mathrm{e}-\mathrm{e})}$ have values that are close to $10390 \mathrm{ppm}$, where $\sigma^{\text {atom(e-e) }}\left(\mathrm{At}^{-}\right) \cong 10563 \mathrm{ppm}$. Then, we 226 propose a model that includes all of these considerations 227

$$
\boldsymbol{\sigma}_{Y}^{\mathrm{M}-\mathrm{V}}=\frac{m_{\mathrm{p}}}{g_{Y}} \boldsymbol{M}_{Y} \otimes \boldsymbol{I}+\boldsymbol{\sigma}_{Y}^{\text {atom }}+\frac{1}{2 c}\left(\boldsymbol{\nu}_{Y}^{\mathrm{S}}-\boldsymbol{\nu}_{Y}^{\text {atom, } \mathrm{S}}\right)
$$

For linear molecules this equation is written as

$$
\begin{aligned}
\sigma_{\perp, Y}^{\mathrm{M}-\mathrm{V}} & =\frac{m_{\mathrm{p}} I}{g_{Y}} M_{\perp, Y}+\sigma_{Y}^{\text {atom }}+\frac{1}{2 c}\left(\nu_{\perp, Y}^{\mathrm{S}}-\nu_{\perp, Y}^{\text {atom }, \mathrm{S}}\right) \\
\sigma_{\|, Y}^{\mathrm{M}-\mathrm{V}} & =\sigma_{Y}^{\text {atom }}+\frac{1}{2 c}\left(\nu_{\|, Y}^{\mathrm{S}}-\nu_{\|, Y}^{\text {atom }, \mathrm{S}}\right)
\end{aligned}
$$

Our M-V model has a few important advantages: (i) the 231 restrictions of considering only the SD and (e-e) parts of $\boldsymbol{\nu}_{Y}^{\mathrm{S}}$ are 232 not needed any more; (ii) from calculations we find that $\nu_{Y}^{\mathrm{S}(\mathrm{e}-\mathrm{p})} 233$ $\cong \boldsymbol{\nu}_{Y}^{\text {atom }, \mathrm{S}(\mathrm{e}-\mathrm{p})}$ and $\boldsymbol{\nu}_{Y}^{\mathrm{S}-\mathrm{SF}(\mathrm{e}-\mathrm{e})} \cong \boldsymbol{\nu}_{Y}^{\text {atom,S-SF }(\mathrm{e}-\mathrm{e})}$. Therefore, $\boldsymbol{\nu}_{Y}^{\mathrm{S}}-\boldsymbol{\nu}_{Y}^{\text {atom, } \mathrm{S}} 234$ $\cong \boldsymbol{\nu}_{Y}^{\mathrm{S} \text {-SD(e-e) }}-\boldsymbol{\nu}_{Y}^{\text {atom,S-SD(e-e) }}$ and (iii) the application of the linear 235 response within the elimination of small components (LRESC) 236 model to $\frac{1}{2 c}\left(\boldsymbol{\nu}_{Y}^{\mathrm{S}}-\boldsymbol{\nu}_{Y}^{\text {atom, } \mathrm{S}}\right)$ gives, as the leading order ${ }_{237}$ contribution, $\frac{1}{2} \boldsymbol{\sigma}_{Y}^{\text {SO-S }}$.

In Table 1 we compare results of the (e-e) contributions to 239 the shieldings of nuclei in ionized free atoms (except for Xe) 240 and the differences between four-component calculations of 241 shieldings of the nucleus in molecules, SR constants, and the S- 242 SD terms. Also, the differences when replacing the S-SD terms 243 by $\nu_{Y}^{S(\mathrm{e}-\mathrm{e})}-\boldsymbol{\nu}_{Y}^{\text {atom, } S(\mathrm{e}-\mathrm{e})}$ are shown. We see that the values of 244 t e n s or e lements of 245 $\boldsymbol{\sigma}_{Y}^{(\mathrm{e}-\mathrm{e})}-\frac{m_{\mathrm{p}}}{g_{Y}} \boldsymbol{M}_{Y}^{(\mathrm{e}-\mathrm{e})} \otimes \boldsymbol{I}-\frac{1}{2 c}\left(\boldsymbol{\nu}_{Y}^{\mathrm{S}(\mathrm{e}-\mathrm{e})}-\boldsymbol{\nu}_{Y^{-}}^{\text {atom, } \mathrm{S}(\mathrm{e}-\mathrm{e})}\right)$ are closer to $\sigma_{Y}{ }^{-}$tom $($e - e $)$than the elements of 246 $\boldsymbol{\sigma}_{Y}^{(\mathrm{e}-\mathrm{e})}-\frac{m_{\mathrm{p}}}{g_{Y}} \boldsymbol{M}_{Y}^{(\mathrm{e}-\mathrm{e})} \otimes \boldsymbol{I}-\frac{1}{2 c} \boldsymbol{\nu}_{Y}^{\mathrm{S}-\mathrm{SD}(\mathrm{e}-\mathrm{e})}$. So, the $\mathrm{M}-\mathrm{V}$ model is more accurate than the M-IV one.

What is the behavior of the M-V model in the NR limit? We 248 scaled the speed of light several times in all calculations of the 249 (e-e) contributions, and its results are summarized as 
Table 1. Comparison of $\sigma^{\text {atom(e-e) }}\left(Y^{-}\right)$with the Difference among the Calculated (e-e) Parts of Shielding, the SR Constant and the S-SD Term, or the $\nu^{S}-\nu^{\text {atom,S }}$ One ${ }^{a}$

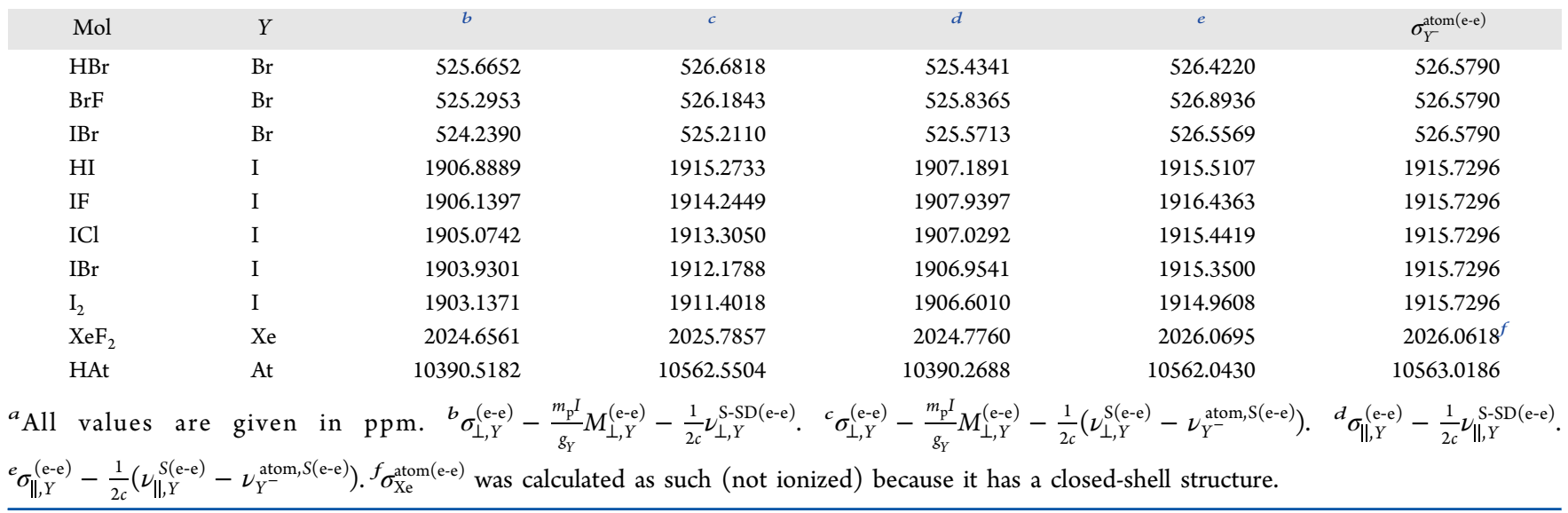

Table 2. Four-Component Calculations of the Isotropic Parameters, $M, \sigma^{\text {atom }}, \nu^{\mathrm{S}}, \nu^{\text {atom, },}$, and $\sigma$, at RPA Level of Approach ${ }^{a, b}$

\begin{tabular}{|c|c|c|c|c|c|c|c|}
\hline Mol & $Y$ & $\frac{2}{3} \frac{m_{\mathrm{p}} I}{g_{\mathrm{Y}}} M_{\perp, Y}$ & $\sigma_{Y}^{\text {atom }}$ & $\frac{1}{2 c} \nu_{Y}^{\text {S,iso }}$ & $\frac{1}{2 c} \nu_{Y^{-}}^{\text {atom }, \mathrm{S}}$ & $\sigma_{Y}^{M-V, \text { iso }}$ & $\sigma_{Y}^{\text {iso }}$ \\
\hline $\mathrm{HF}$ & $\mathrm{F}$ & -57.9462 & 480.3801 & 2.0197 & 2.0608 & 422.3927 & 415.8361 \\
\hline $\mathrm{ClF}$ & $\mathrm{F}$ & 210.9556 & 480.3801 & 1.7950 & 2.0608 & 691.0699 & 683.9108 \\
\hline $\mathrm{BrF}$ & $\mathrm{F}$ & 291.9238 & 480.3801 & -6.9508 & 2.0608 & 763.2923 & 756.7222 \\
\hline IF & $\mathrm{F}$ & $\begin{array}{c}375.8094 \\
{[375.8150]}\end{array}$ & $\begin{array}{c}480.3801 \\
{[482.3759]}\end{array}$ & $\begin{array}{c}-56.3174 \\
{[-56.4510]}\end{array}$ & $\begin{array}{c}2.0608 \\
{[1.9266]}\end{array}$ & $\begin{array}{c}797.8113 \\
{[799.8132]}\end{array}$ & $\begin{array}{c}790.7507 \\
{[793.7221]}\end{array}$ \\
\hline $\mathrm{XeF}_{2}$ & $\mathrm{~F}$ & $\begin{array}{c}-31.9061 \\
{[-31.9027]}\end{array}$ & $\begin{array}{c}480.3801 \\
{[482.3759]}\end{array}$ & $\begin{array}{c}-2.2784 \\
{[-2.4125]}\end{array}$ & $\begin{array}{c}2.0608 \\
{[1.9266]}\end{array}$ & $\begin{array}{c}444.1348 \\
{[446.1340]}\end{array}$ & $\begin{array}{c}438.4208 \\
{[440.4896]}\end{array}$ \\
\hline $\mathrm{HCl}$ & $\mathrm{Cl}$ & -192.3764 & 1132.2008 & 13.8434 & 13.9431 & 939.7247 & 934.1685 \\
\hline $\mathrm{ClF}$ & $\mathrm{Cl}$ & -1598.3155 & 1132.2008 & 13.2515 & 13.9431 & -466.8064 & -473.7810 \\
\hline $\mathrm{ICl}$ & $\mathrm{Cl}$ & 25.7220 & 1132.2008 & -7.3600 & 13.9431 & 1136.6197 & 1129.3206 \\
\hline $\mathrm{HBr}$ & $\mathrm{Br}$ & $\begin{array}{c}-482.9111 \\
{[-482.9190]}\end{array}$ & $\begin{array}{c}3355.2831 \\
{[3439.9540]}\end{array}$ & $\begin{array}{c}130.3628 \\
{[121.1226]}\end{array}$ & $\begin{array}{c}133.3157 \\
{[124.0756]}\end{array}$ & $\begin{array}{c}2869.4190 \\
{[2954.0819]}\end{array}$ & $\begin{array}{c}2865.2450 \\
{[2948.7345]}\end{array}$ \\
\hline $\mathrm{BrF}$ & $\mathrm{Br}$ & -3569.1870 & 3355.2831 & 94.3739 & 133.3157 & -252.8456 & -258.1816 \\
\hline $\mathrm{IBr}$ & $\mathrm{Br}$ & -388.0969 & 3355.2831 & 156.9831 & 133.3157 & 2990.8537 & 2983.1190 \\
\hline HI & I & $\begin{array}{c}-902.1302 \\
{[-902.1781]}\end{array}$ & $\begin{array}{c}6406.4582 \\
{[6803.6769]}\end{array}$ & $\begin{array}{c}511.0723 \\
{[495.7096]}\end{array}$ & $\begin{array}{c}541.2102 \\
{[525.8491]}\end{array}$ & $\begin{array}{c}5474.1901 \\
{[5871.3594]}\end{array}$ & $\begin{array}{c}5469.7790 \\
{[5866.5947]}\end{array}$ \\
\hline IF & I & $\begin{array}{c}-5630.2973 \\
{[-5630.9135]}\end{array}$ & $\begin{array}{c}6406.4582 \\
{[6803.6769]}\end{array}$ & $\begin{array}{c}278.0488 \\
{[262.6546]}\end{array}$ & $\begin{array}{c}541.2102 \\
{[525.8491]}\end{array}$ & $\begin{array}{c}512.9994 \\
{[909.5689]}\end{array}$ & $\begin{array}{c}507.5656 \\
{[903.8078]}\end{array}$ \\
\hline $\mathrm{ICl}$ & I & -2827.5506 & 6406.4582 & 294.2667 & 541.2102 & 3331.9641 & 3326.0317 \\
\hline $\mathrm{IBr}$ & I & -2184.7155 & 6406.4582 & 310.5022 & 541.2102 & 3991.0347 & 3984.5477 \\
\hline $\mathrm{I}_{2}$ & I & -1236.6301 & 6406.4582 & 494.2027 & 541.2102 & 5122.8205 & 5112.9888 \\
\hline $\mathrm{XeF}_{2}$ & $\mathrm{Xe}$ & $\begin{array}{c}-3609.8499 \\
{[-3609.9578]}\end{array}$ & $\begin{array}{c}6619.4386^{c} \\
{[7020.7966]^{c}}\end{array}$ & $\begin{array}{c}562.4590 \\
{[546.6925]}\end{array}$ & $\begin{array}{c}570.4629^{c} \\
{[554.6929]^{c}}\end{array}$ & $\begin{array}{c}3001.5848 \\
{[3402.8383]}\end{array}$ & $\begin{array}{c}2999.2927 \\
{[3400.2130]}\end{array}$ \\
\hline HAt & At & $\begin{array}{c}-146.9260 \\
{[-147.4289]}\end{array}$ & $\begin{array}{c}18778.8819 \\
{[19193.0912]}\end{array}$ & $\begin{array}{c}2797.5863 \\
{[2761.4493]}\end{array}$ & $\begin{array}{c}3662.3453 \\
{[3626.2449]}\end{array}$ & $\begin{array}{c}17767.1969 \\
{[18180.8666]}\end{array}$ & $\begin{array}{c}17762.6035 \\
{[18175.7059]}\end{array}$ \\
\hline
\end{tabular}

${ }^{a} \mathrm{RKB}$ prescription was used in all calculations, but between brackets are the UKB calculations. ${ }^{b}$ All values in ppm. ${ }^{c} \sigma^{\text {atom }}(\mathrm{Xe})$ and $\nu^{\text {atom, }}(\mathrm{Xe})$ were calculated as such (not $\mathrm{Xe}^{-}$) because it has a closed-shell electronic structure.

251 (1) For all studied systems, the SD values of $\boldsymbol{\sigma}_{Y}, \boldsymbol{\sigma}_{Y}^{\text {atom }}, \boldsymbol{M}_{Y}^{\mathrm{L}}, \boldsymbol{M}_{Y}^{\mathrm{S}}$ 252 (and therefore also $\nu_{Y}^{\mathrm{L}}$ and $\boldsymbol{\nu}_{Y}^{\mathrm{S}}$ ), and $\boldsymbol{\nu}_{Y}^{\text {atom,S }}$ go to zero as $c$ scales 253 to $\infty$. This behavior is fine because they arise only within the 254 relativistic domain.

255 (2) The SF contribution to $\boldsymbol{M}_{Y}^{S}, \boldsymbol{\nu}_{Y}^{S}$, and $\boldsymbol{\nu}_{Y}^{\text {atom, } S}$ also goes to 256 zero as $c \rightarrow \infty$.

257 Some remarks concerning the isotropic contributions of both 258 properties need to be made. As was pointed out in ref 7, the M259 II and M-III models match each other when isotropic values of 260 shieldings of nuclei in linear molecules are considered. This 261 occurs because $\sigma_{Y}^{\text {SO-S,iso }}$ is zero for linear molecules, ${ }^{22}$ but $\frac{1}{2 c} \nu_{Y}^{\mathrm{S}-\mathrm{SD}(\mathrm{e}-\mathrm{e}) \text {, iso }}$ and $\frac{1}{2 c}\left(\nu_{Y}^{\mathrm{S}(\mathrm{e}-\mathrm{e}), \text { iso }}-\nu_{Y}^{\text {atom, } \mathrm{S}(\mathrm{e}-\mathrm{e})}\right)$ are not zero.
In Table 2 we show the values of 262 t2 $\sigma_{Y}^{\mathrm{M}-\mathrm{V}, \text { iso }}=\frac{2}{3} \frac{m_{\mathrm{p}} I}{g_{Y}} M_{\perp, Y}+\sigma_{Y^{-}}^{\text {atom }}+\frac{1}{2 c}\left(\nu_{Y}^{\mathrm{S} \text {,iso }}-\nu_{Y^{-}}^{\text {atom,S }}\right)$.

In addition, a good agreement between $\sigma_{\perp, Y}^{\mathrm{M}-\mathrm{V}(\mathrm{e}-\mathrm{e})}$ and $\sigma_{\perp, Y}^{(\mathrm{e}-\mathrm{e})}$ and 263 also between $\sigma_{\|, Y}^{\mathrm{M}-\mathrm{V}(\mathrm{e}-\mathrm{e})}$ and $\sigma_{\|, Y}^{(\mathrm{e}-\mathrm{e})}$ is found. The differences are of 264 $<1 \mathrm{ppm}$ for the parallel tensor elements of all nuclei in all 265 compounds $(<0.8 \%$ of difference in all studied cases, except for 266 $\mathrm{F}$ in $\mathrm{HF}$ where the difference is of $0.1365 \mathrm{ppm}$ against $\sigma_{\|, \mathrm{F}}^{(\mathrm{e} e)}=267$ $8.2395 \mathrm{ppm}$ and $\mathrm{Br}$ in $\mathrm{BrF}$, where the difference is of 0.3146268 ppm while $\left.\sigma_{\|, \mathrm{Br}}^{(\mathrm{e}-\mathrm{e})}=2.7290 \mathrm{ppm}\right)$. For the perpendicular 269 elements, the greatest difference in their (e-e) contributions 270 occurs for $\mathrm{I}_{2}$, and it is $\sim 4.3278 \mathrm{ppm}$ ( $4.5 \%$ of difference with 271 respect to $\sigma_{\perp, I}^{(\mathrm{e}-\mathrm{e})}$, which is $\left.96.0347 \mathrm{ppm}\right)$; all other perpendicular 272 
273 elements give a percentage difference of $<0.75 \%$. Therefore, the 274 main differences between $\sigma_{Y}^{M-V \text {,iso }}$ and $\sigma_{Y}^{\text {iso }}$ in Table 2 are due to 275 their (e-p) contributions.

276 In the special case of $\mathrm{XeF}_{2}$, it is well known that there is a 277 difference between $\sigma_{\|, Y}$ of the nuclei in the molecule and $\sigma_{Y}^{\text {atom }}$ 278 of that nuclei in free atoms $(Y=\mathrm{Xe}$ and $\mathrm{F})$. This was an 279 indication that Flygare's relation is broken when heavy atoms 280 are considered. Flygare's NR relation states that for a nucleus $Y$ 281 in a linear molecule, $\sigma_{\|, Y}^{\mathrm{NR}-\text { para }}$ is zero and $\sigma_{Y}^{\text {atom,NR-para }}$ is also zero. 282 In addition, it holds that $\sigma_{\|, Y}^{\mathrm{NR}-\mathrm{dia}} \cong \sigma_{Y}^{\text {atom,NR-dia }}=\sigma_{Y}^{\text {atom,NR }}$.

283 What happens within the relativistic domain? We shall 284 analyze the (e-e) and (e-p) contributions to the shieldings. The 285 calculations of the (e-p) part at RPA level give: $\sigma_{\|}^{(\mathrm{e}-\mathrm{p})}\left(\mathrm{Xe}_{\mathrm{XeF}}\right)$ $286=4599.7948 \mathrm{ppm}$, whereas $\sigma^{\text {atom }(\mathrm{e}-\mathrm{p})}(\mathrm{Xe})=4593.3768 \mathrm{ppm}$. It 287 is seen that there is only a small difference between them, that 288 is, $6.4180 \mathrm{ppm}$. For fluorine a difference of $18.9435 \mathrm{ppm}$ is 289 found. The differences in both cases are probably due to 290 quadrupole terms. ${ }^{11}$

291 The relativistic (e-e) contribution has a large difference with 292 its NR counterpart. Within the relativistic regime $\sigma_{Y}^{\text {atom(e-e) }}$ and $293 \sigma_{\|, Y}^{(\mathrm{e}-\mathrm{e})}$ are both different from zero. In the case of $\mathrm{Xe}$ in $\mathrm{XeF}_{2}$, $294 \sigma_{\|}^{(\mathrm{e}-\mathrm{e})}(\mathrm{Xe})=1388.2505 \mathrm{ppm}$, while $\sigma^{\text {atom }(\mathrm{e}-\mathrm{e})}(\mathrm{Xe})=2026.0618$ $295 \mathrm{ppm}$, being its difference of $-637.8113 \mathrm{ppm}$. We assume that 296 the mechanism responsible for this difference is related with $297 \nu_{\|}^{S(\mathrm{e}-\mathrm{e})}-\nu^{\text {atom,S(e-e) }}$. Its RPA value for $\mathrm{Xe}$ in $\mathrm{XeF}_{2}$ and the atomic $298 \mathrm{Xe}$ is $-637.8190 \mathrm{ppm}$. In the case of fluorine in $\mathrm{XeF}_{2}$ the 299 difference among $\sigma_{\|}^{(\mathrm{e} e \mathrm{e})}(\mathrm{F})$ and $\sigma^{\text {atom(e-e) }}\left(\mathrm{F}^{-}\right)$is $19.3646 \mathrm{ppm}$, 300 whereas $\frac{1}{2 c} \nu_{\|}^{\mathrm{S}(\mathrm{e}-\mathrm{e})}\left(\mathrm{F}: \mathrm{XeF}_{2}\right)-\frac{1}{2 c} \nu^{\text {atom, } \mathrm{S}(\mathrm{e}-\mathrm{e})}\left(\mathrm{F}^{-}\right)$is $19.5146 \mathrm{ppm}$. 301 Therefore, $\sigma_{\|}\left(\mathrm{Xe}: \mathrm{XeF}_{2}\right)-\sigma^{\text {atom }}(\mathrm{Xe}) \cong \nu_{\|}^{\mathrm{S}(\mathrm{e}-\mathrm{e})}-\nu^{\text {atom, } \mathrm{S}(\mathrm{e}-\mathrm{e})}$, and 302 this is because the $(\mathrm{e}-\mathrm{p})$ parts of $\sigma_{\|}^{(\mathrm{e}-\mathrm{p})}$ and $\sigma^{\text {atom(e-p) }}$ are very 303 close each other.

304 To summarize, we have shown here that the best of our 305 relativistic Flygare-like models must include new four306 component terms: $\nu^{\mathrm{S}}$ and $\nu^{\text {atom, } \mathrm{S}}$. These terms are more general 307 than the previously used two-component SO-S term. This one 308 can be obtained as the leading order relativistic correction of 309 the LRESC expansion of $\nu_{Y}^{\mathrm{S}(\mathrm{e}-\mathrm{e})}-\nu_{Y}^{\text {atom, } \mathrm{S}(\mathrm{e}-\mathrm{e})}$

310 The new M-V model is our best to obtain the absolute value 311 of $\sigma$ in light- and heavy-atom-containing molecules. We applied 312 it to a family of $X Y$ and $\mathrm{HX}(X, Y=\mathrm{F}, \mathrm{Cl}, \mathrm{Br}, \mathrm{I}, \mathrm{At})$ linear 313 molecules, reaching accurate fittings with benchmark values of $314 \sigma$.

315 In addition we answered the inquiry first proposed in 2004 316 by the work of Wasylishen and coauthors concerning the large 317 difference between the parallel component of the NMR 318 shielding tensor of $\mathrm{Xe}$ in $\mathrm{XeF}_{2}$ and that of the $\mathrm{Xe}$ free atom. 319 It is almost completely explained through the introduction of 320 the new $\nu^{\mathrm{S}}$ term.

\section{AUTHOR INFORMATION}

\section{Corresponding Author}

323 *E-mail: gaaucar@conicet.gov.ar.

\section{ORCID}

325 Gustavo A. Aucar: 0000-0003-2547-2330

326 Notes

327 The authors declare no competing financial interest.

\section{$328 \square$ ACKNOWLEDGMENTS}

329 We acknowledge partial support from CONICET (Grant PIP 330 112-20130100361) and FONCYT (Grant PICT2012-1214).

\section{REFERENCES}

331

(1) Flygare, W. H. Magnetic Interactions in Molecules and an 332 Analysis of Molecular Electronic Charge Distribution from Magnetic 333 Parameters. Chem. Rev. 1974, 74, 653-687.

334

(2) Forgeron, M. A. M.; Wasylishen, R. E.; Penner, G. H. 335 Investigation of Magnetic Shielding in Xenon Difluoride Using 336 Solid-State NMR Spectroscopy and Relativistic Density Functional 337 Theory. J. Phys. Chem. A 2004, 108, 4751-4758.

(3) Aucar, I. A.; Gomez, S. S.; Ruiz de Azúa, M. C.; Giribet, C. G. 339 Theoretical Study of the Nuclear Spin-Molecular Rotation Coupling 340 for Relativistic Electrons and Non-Relativistic Nuclei. J. Chem. Phys. 341 2012, 136, 204119.

342

(4) Xiao, Y.; Liu, W. Body-Fixed Relativistic Molecular Hamiltonian 343 and its Application to Nuclear Spin-Rotation Tensor. J. Chem. Phys. 344 2013, 138, 134104.

345

(5) Malkin, E.; Komorovsky, S.; Repisky, M.; Demissie, T. B.; Ruud, 346 K. The Absolute Shielding Constants of Heavy Nuclei: Resolving the 347 Enigma of the ${ }^{119}$ Sn Absolute Shielding. J. Phys. Chem. Lett. 2013, 4, 348 459-463.

349

(6) Xiao, Y.; Zhang, Y.; Liu, W. New Experimental NMR Shielding 350 Scales Mapped Relativistically from NSR: Theory and Application. J. 351 Chem. Theory Comput. 2014, 10, 600-608.

352

(7) Aucar, I. A.; Gomez, S. S.; Giribet, C. G.; Aucar, G. A. Toward an 353 Absolute NMR Shielding Scale Using the Spin-Rotation Tensor within 354 a Relativistic Framework. Phys. Chem. Chem. Phys. 2016, 18, 23572- 355 23586.

356

(8) Ramsey, N. F. Magnetic Shielding of Nuclei in Molecules. Phys. 357 Rev. 1950, 78, 699-703.

(9) Flygare, W. H. Spin-Rotation Interaction and Magnetic Shielding 359 in Molecules. J. Chem. Phys. 1964, 41, 793-800.

(10) Flygare, W. H.; Goodisman, J. Calculation of Diamagnetic 361 Shielding in Molecules. J. Chem. Phys. 1968, 49, 3122-3125. 362

(11) Gierke, T. D.; Flygare, W. H. Empirical Evaluation of the 363 Individual Elements in the Nuclear Diamagnetic Shielding Tensor by 364 the Atom Dipole Method. J. Am. Chem. Soc. 1972, 94, 7277-7283. 365

(12) Aucar, I. A.; Gomez, S. S.; Melo, J. I.; Giribet, C. G.; Ruiz de 366 Azúa, M. C. Theoretical Study of the Nuclear Spin-Molecular Rotation 367 Coupling for Relativistic Electrons and Non-Relativistic Nuclei. II. 368 Quantitative Results in HX (X = H, F, Cl, Br, I) Compounds. J. Chem. 369 Phys. 2013, 138, 134107.

(13) Aucar, G. A.; Romero, R. H.; Maldonado, A. F. Polarization 371 Propagators: A Powerful Theoretical Tool for a Deeper Understanding 372 of NMR Spectroscopic Parameters. Int. Rev. Phys. Chem. 2010, 29, 1- 373 64.

(14) Gomez, S. S.; Aucar, G. A. Relativistic Effects on the Nuclear 375 Magnetic Resonance Shielding of FX (X = F, Cl, Br, I, and At) 376 Molecular Systems. J. Chem. Phys. 2011, 134, 204314.

(15) Dyall, K. G. An Exact Separation of the Spin-Free and Spin- 378 Dependent Terms of the Dirac-Coulomb-Breit Hamiltonian. J. Chem. 379 Phys. 1994, 100, 2118.

(16) Aucar, I. A. Efectos de Átomos Pesados en Parámetros 381 Magnéticos Moleculares. Ph.D. Thesis, 2015.

(17) Saue, T., Visscher, L., Aa. Jensen, H. J., Bast, R.; et al. DIRAC, a 383 Relativistic Ab Initio Electronic Structure Program, Release DIRAC14, 384 2014. http://www.diracprogram.org.

(18) Handbook of Chemistry, 3rd ed.; Maruzen Company: Tokyo, 386 1984; Vol. II.

(19) Huber, K. P.; Herzberg, G. Constants of Diatomic Molecules; 388 Nostrand Reinhold: New York, 1979.

(20) Dunning, T. H. Gaussian Basis Sets for Use in Correlated 390 Molecular Calculations. I. The Atoms Boron Through Neon and 391 Hydrogen. J. Chem. Phys. 1989, 90, 1007-1023.

(21) Dyall, K. G. Relativistic Quadruple-Zeta and Revised Triple-Zeta 393 and Double-Zeta Basis Sets for the 4p, 5p, and 6p Elements. Theor. 394 Chem. Acc. 2006, 115, 441-447.

(22) Ruiz de Azúa, M. C.; Giribet, C. G.; Melo, J. I. NMR Nuclear 396 Magnetic Shielding Anisotropy of Linear Molecules within the Linear 397 Response within the Elimination of the Small Component Approach. J. 398 Chem. Phys. 2011, 134, 034123. 